\title{
Crystal structure and morphology of $\beta$-HMX in acetone: A molecular dynamics simulation and experimental study
}

\author{
JUN TAO* and XIAOFENG WANG \\ The Second Department, Xi' an Modern Chemistry Research Institute, Xi'an 710065, China \\ Email: taojun4712230@126.com
}

MS received 20 November 2016; revised 9 February 2017; accepted 12 February 2017

\begin{abstract}
Single crystals of $\beta$-Cyclotetramethylene tetranitramine (HMX) were prepared by the solvent evaporation method. The structure was then determined using infrared spectroscopy and single crystal X-ray diffraction. The modified attachment energy (AE) model was used to predict the morphologies of $\beta$-HMX in vacuum and in acetone. The morphology and sensitivity of HMX before and after recrystallization were characterized. The results of calculation showed that the (011) and (110) surfaces of $\beta$-HMX are of great morphological importance. The predicted $\beta$-HMX morphology agreed qualitatively with the SEM result. The sensitivity results show that recrystallization in acetone can effectively reduce the impact and friction sensitivities of $\beta$-HMX.
\end{abstract}

Keywords. $\quad \beta$-HMX; single crystal; attachment energy model; crystal morphology; impact sensitivity; friction sensitivity.

\section{Introduction}

As an excellent high explosive, cyclotetramethylene tetranitramine (HMX) is used for military purposes, as a component of plastic-bonded explosives, as a component of rocket propellants, and as a high explosive buster charge. It has advantage in high density of charges that provide for a higher rate of detonation, for more efficient application..$^{1-3}$ The detonation performance and specifically the sensitivity of HMX not only have a relationship with the morphology $y^{4-7}$ of the HMX crystals, but also depend on the defects ${ }^{8}$ present in the crystals and the crystal phase. ${ }^{9}$ The attachment stability of growth units (HMX molecules) on each surface of the crystal affects the crystal morphology. In addition, the crystal morphology is also connected with the physical and chemical conditions during growth. ${ }^{10-12}$ Therefore, the crystal structure should be closely linked with the growth conditions in HMX crystal morphology research.

At the present time, crystal growth theories and crystal morphology predictions of explosives are being investigated with growing interest. ${ }^{13-16}$ ter Horst et al., added a $\gamma$-butyrolactone layer to (200) and (210) crystal surfaces of RDX. They found that the (210) face is morphologically more important than the (200) face, the molecular dynamics simulation results agree well with the observed experimental RDX morphology

\footnotetext{
*For correspondence
}

grown in the solvent $\gamma$-butyrolactone. ${ }^{17}$ Shi et al., predicted the growth morphology of 2,6-diamino-3,5dinitropyridine-1-oxide (ANPyO) in vacuum and in trifluoroacetic acid solvent by the corrected attachment energy (AE) model. The diffusion coefficient of trifluoroacetic acid molecules on the crystal growth faces has also been analyzed, and the results will be useful for the formulation design of ANPyO.$^{18}$ Liu et al., studied the crystal morphologies of 3,4-bis(3-nitrofurazan-4yi) furoxan (DNTF) ${ }^{19}$ and 1,1-diamino-2,2-dinitroethylene $(\text { FOX }-7)^{20}$ using the AE model. The impact and friction sensitivities were also tested and discussed for different crystal morphologies. However, as an excellent high explosive, there is little study on the crystal growth theories ${ }^{21,22}$ and crystal morphology predictions of HMX.

In this study, HMX single crystals were prepared and the single crystal structure with low $\mathrm{R}$ factor was obtained. In order to study the effect of the interaction between solvent molecules and crystal surfaces on the crystal morphology at the micro scale, the adsorption energy and crystal morphology of HMX in vacuum and acetone were studied by the AE model and molecular dynamics (MD) method (the obtained single crystal being used as the input). The intrinsic and extrinsic factors controlling the morphology were analyzed from the point of view of HMX polarity and the solvent interaction. The single crystal structure can be used as input for molecular simulations for HMX and polymer bonded explosives (PBXs) containing HMX. The morphology 
results can provide a theoretical basis for the selection of solvent in the crystallization process of HMX.

\section{Experimental and computational methods}

\subsection{Preparation and structure determination of $H M X$ single crystal}

$\beta-\mathrm{HMX}, \mathrm{C}_{4} \mathrm{H}_{8} \mathrm{~N}_{8} \mathrm{O}_{8}$, M.W. $=296.18 \mathrm{~g} \cdot \mathrm{mol}^{-1}$, is a colorless and transparent crystal. A single crystal of $\beta$-HMX was prepared by the solvent evaporation method. Herein, HMX 1 and HMX 2 correspond to the crystals before and after recrystallization from acetone. A crystal, approximately $0.4 \times 0.4 \times 0.4 \mathrm{~mm}^{3}$, was used for this experiment, and the crystal type being identified from its infrared spectrum. For this, the scattering angles $(2 \theta)$ of the most intense reflections having scattering angles in the range of $3^{\circ}$ to $65^{\circ}$ were measured using an automated Rigaku AFC-6 4-circle diffractometer with $\mathrm{MoK} \alpha$ radiation monochromated using a pyrolytic graphite plate.

\subsection{Crystal morphology predicted by AE model}

The AE model of crystal growth was developed on the basis of the periodic bond -chain (PBC) model. The PBC model assumes that, (i) the time needed for bonding to the crystal surface is inversely proportional to the bonding energy, (ii) the crystal growth rate is proportional to the bonding energy, (iii) the crystal is composed of $\mathrm{PBC}$, and (iv) the fastest crystal growth direction is the direction of the strongest bonds. In the AE model, by analyzing the center-to-plane distance assigned to the dominant crystal surface, crystal morphologies can be obtained, and the center-to-plane distance assigned relates to the relative growth rate. The attachment energy $\left(E_{\text {att }}\right)$ is the energy released by adding a growth slice to a growing crystal surface. ${ }^{19,23} E_{\text {att }}$ can be calculated as follows:

$$
E_{\text {att }}=E_{\text {latt }}-E_{\text {slice }}
$$

Here, the slice energy ( $\left.E_{\text {slice }}\right)$ is the energy released by growing a crystal slice with a thickness of $d_{\mathrm{hkl}}$, and the attachment energy $\left(E_{\text {att }}\right)$ being the energy released by attaching a slice to the crystal surface. The sum of the two is the lattice energy $\left(E_{\mathrm{latt}}\right) . R_{\mathrm{hkl}}$ is the growth rate of (hkl) face of HMX in vacuum and is proportional to the attachment energy of the crystal.

$$
R_{\mathrm{hkl}} \propto E_{\mathrm{att}}
$$

As the attachment energies for acetone molecules on the different growth faces are different, acetone is important in the crystal growth process. The stronger the adsorption, the easier is the solvent layer formation on the crystal-solvent interface. Acetone will limit the growth of the crystal. Defining $E_{\text {int }}$ as the crystal-acetone interaction energy, it can be calculated using the following formula:

$$
E_{\text {int }}=E_{\text {tot }}-E_{\text {surf }}-E_{\text {solv }}
$$

Here, $E_{\text {tot }}$ is the sum of crystal surface energy and acetone layer energy, $E_{\text {surf }}$ is the energy of crystal surface, $E_{\text {solv }}$ is the energy of the acetone layer.

Considering the influence of the acetone layer, the calibration factor $E_{\mathrm{s}}$ for vacuum attachment energy is introduced, which describes the binding energy of solvent on the (hkl) face of HMX crystal. It can be calculated as follows: ${ }^{19}$

$$
E_{\mathrm{s}}=E_{\text {int }} \times \frac{A_{\text {acc }}}{A_{\text {cell }}}
$$

Here, $A_{\text {acc }}$ is the acetone-accessible area of the crystal face in the unit cell. ${ }^{14,19} A_{\text {cell }}$ is the total crystal face area along the (hkl) plane.

The acetone attachment energy can be described using the formula:

$$
E_{\mathrm{att}}^{\prime}=E_{\mathrm{att}}-E_{\mathrm{s}}
$$

The growth rate of each crystal face $\left(R_{h k l}^{\prime}\right)$ is proportional to the acetone attachment $\left(E_{a t t}^{\prime}\right)$ energy of the crystal (as shown in eq. 6), which is given by Hartman in the modified morphology theory. ${ }^{24}$

$$
R_{\mathrm{hkl}}^{\prime} \propto\left|E_{\mathrm{att}}^{\prime}\right|
$$

\subsection{Molecular dynamics simulation}

The $\beta$-HMX original cell was built according to the single crystal X ray diffraction results and the forceit module ${ }^{25}$ was applied to optimize the original structure. Then, the growth morphology in vacuum was predicted by the AE model, to obtain the main growth faces (hkl). A $3 \times 3 \times 3$ super cell was built based on the optimized original cell and cutting crystal along (011), (110), (020), (11-1) and (101) faces, respectively.

A $20 \AA$ Á vacuum layer was added on each crystalline surface of $\beta$-HMX. Then, the solvent-accessible area was calculated using the Atom Volumes and Surfaces tool of the MS software, ${ }^{26}$ which creates a field that can characterize the geometry and solvent interaction of an atomistic structure in a variety of ways. ${ }^{27}$ For the Connolly task in the Atom Volumes and Surfaces tool, there is a field whose value at each point in space corresponds to the depth in the nearest Connolly probe of a given radius, as it rolls over the van der Waals surface of the atomistic structure. ${ }^{28}$

The amorphous cell module was applied to build the solvent layer containing 200 random acetone molecules with a target density of $0.788 \mathrm{~g} \cdot \mathrm{cm}^{-3}$. The size of the periodic cell for the acetone layer must be consistent with the size of the HMX periodic cell. Further, the Andersen thermostat was applied to carry out geometry optimization and MD simulation in the NPT ensemble for acetone molecules.

The adsorption models for HMX surface-acetone were built from a HMX crystal layer and an acetone layer. The acetone layer was placed along the $\mathrm{c}$ axis on the HMX surface and a vacuum upto a height of $30 \AA$ above the acetone layer. COMPASS force field ${ }^{29}$ was selected. Optimizing the 
initial configuration of HMX-acetone and annealing (300$500 \mathrm{~K}, 10^{4}$ steps) of the HMX-solvent configuration were used to eliminate unreasonable conformations. The configurations were taken after annealing for the MD simulation at constant pressure and temperature (NPT) ensemble at $298 \mathrm{~K}$ and $10^{5} \mathrm{~Pa}$ for $500 \mathrm{ps}$. Andersen thermostat was used. ${ }^{30-33}$

\subsection{Recrystallization experiments}

HMX crystals were recrystallized in acetone by solvent evaporation. Recrystallization experiments were carried out in new $50 \mathrm{~mL}$ volumetric flasks at room temperature. The flasks were sealed with plastic film with several holes and left to stand for about a week to obtain HMX crystals.

\subsection{Sensitivity test}

Chinese GJB772A-97 method 601.1 was used to test the impact sensitivity. ${ }^{34}$ The samples were sandwiched between two smooth hard surfaces and impacted by a drop hammer falling freely from a fixed height. The explosion probability was tested to characterize the impact sensitivity. The weight of the hammer was $10 \mathrm{~kg}$, and that of the explosive sample was $50 \mathrm{mg}$. The experimental device is shown in Figure 1. The drop hammer fell freely from a height of $25 \mathrm{~cm}$. When there was a phenomenon of sound, light, smoke, sample discoloration, traces on the surface of the impact pillar, or smell of the gaseous products of decomposition or explosion, it was judged to be an explosion. Otherwise, there was no explosion. The explosive probability of a set of tests ( 25 samples) was calculated as follows:

$$
P=\frac{X}{25} \times 100 \%
$$

Here, $\mathrm{P}$ is the explosion probability, and $\mathrm{X}$ is the number of explosions for 25 samples.

Friction sensitivity was tested according to Chinese GJB772A-97 method 602.1. The samples were sandwiched between two smooth hard surfaces, loaded at constant pressure and external force of sliding friction. The explosion probability was tested to characterize the friction sensitivity. The WM-1 friction sensitivity instrument was used which is shown in Figure 2. The gauge pressure was $3.92 \mathrm{MPa}$, and

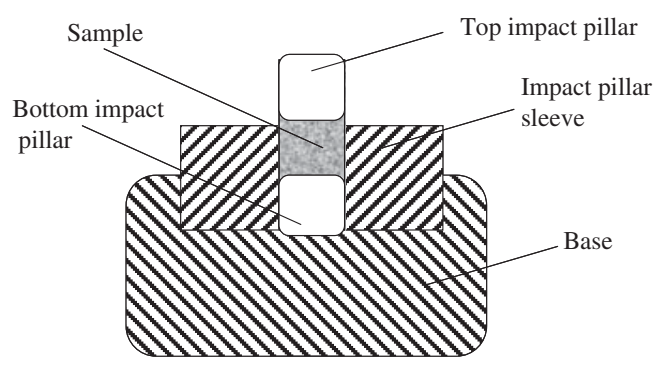

Figure 1. The experimental device for impact explosion test.

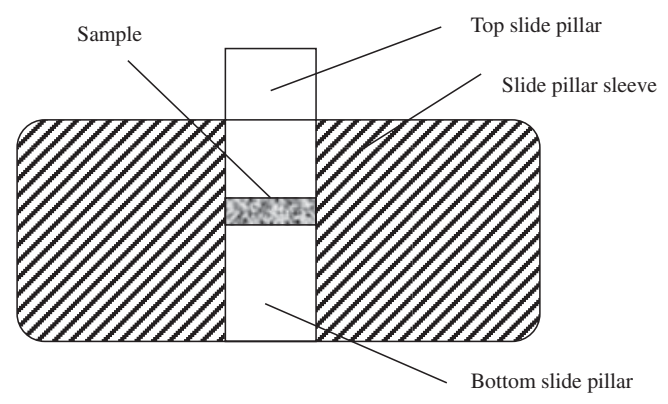

Figure 2. The experimental device for friction test for explosion.

the explosive sample weight was $20 \mathrm{mg}$. The swing angle was set as $90^{\circ}$, and then the impact hammer of the pendulum was released, and the top slide pillar moves a distance. When there was a phenomenon of sound, light, smoke, sample discoloration, traces on the surface of impact pillar, or smell of the gaseous products of decomposition or explosion, it was judged to be an explosion. Otherwise, there was no explosion. The explosive probability of a set of tests ( 25 samples) was calculated by eq. 7 .

\section{Results and Discussion}

\subsection{Single crystal structure}

An infrared spectrum (Figure 3(a)) was taken to determine the crystal type of the prepared HMX sample. There is no peak in the range of $700-750 \mathrm{~cm}^{-1}$, therefore the sample is $\beta$-HMX. ${ }^{35,36}$ There is no phase transition in the preparation process of the single crystal of HMX. The single crystal structure of $\beta$-HMX is shown in Figure 3(b). $\beta$-HMX crystallizes in the orthorhombic space group $\mathrm{P} 2(4) / \mathrm{n}$. The dimensions thus obtained are $\mathrm{a}=6.548(2) \AA, \mathrm{b}=11.041(4) \AA, \mathrm{c}=7.369(3) \AA$, $\beta=102.738(5)^{\circ}, \mathrm{Z}=2, \mathrm{~V}=519.6(3) \AA^{3}$, and the observed density is $D_{\mathrm{c}}=1.893 \mathrm{~g} \cdot \mathrm{cm}^{-3}$. The final leastsquares parameters for the structure of HMX are shown in Table 1.

In order to further study the structure of $\beta$-HMX crystal, the obtained structure was compared with the structure used most widely in molecular simulations (structure B). The identity number of structure B in the CCSD database is OCHTET12, for which $\mathrm{R}$ factor is the lowest in the database. As shown in Table 2, there are differences between structure A and structure B. First, "c" of structure A is $7.369 \AA$, while "c" of structure B is $8.700 \AA$ '; second, " $\beta$ " of structure $\mathrm{A}$ is smaller than structure $\mathrm{B}$; third, structure A and structure B belong to different space groups. Most importantly, the R factor of structure A is smaller than that for structure B, which suggests that the accuracy of structure $\mathrm{A}$ is higher 


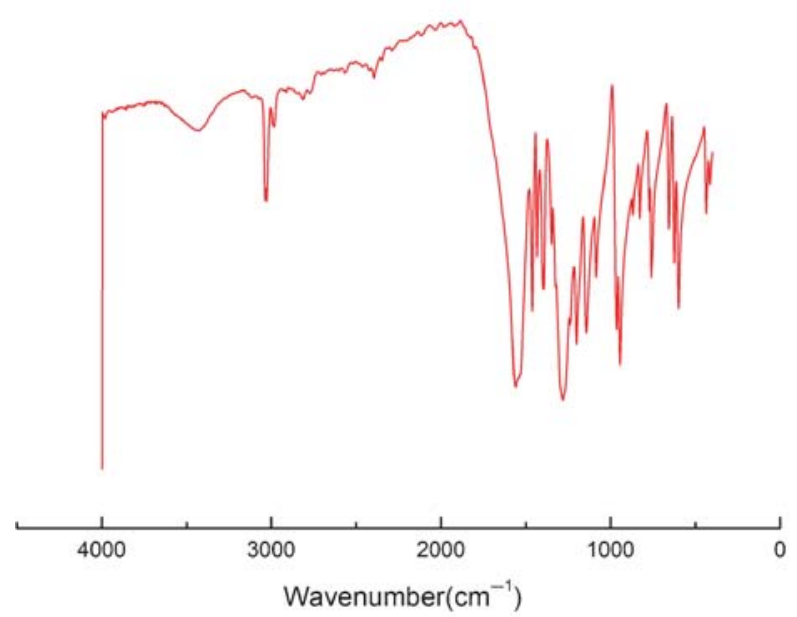

(a)

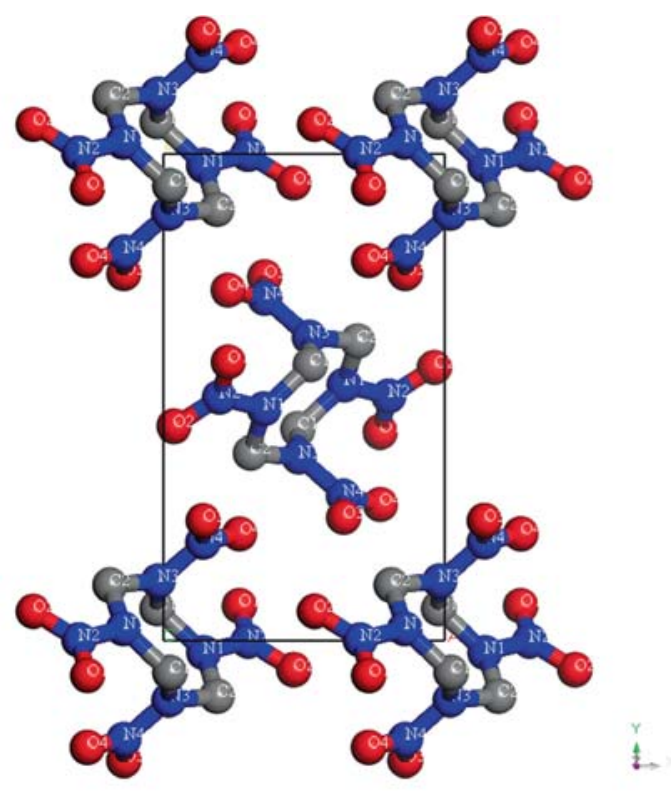

(b)

Figure 3. (a) Infrared spectrum of $\beta$-HMX; (b) Packing of the HMX molecules in crystal lattice.

Table 1. Final least-squares parameters for the structure of HMX.

\begin{tabular}{lcclc}
\hline Atom & $\mathrm{x}$ & $\mathrm{y}$ & \multicolumn{1}{c}{$\mathrm{z}$} & $\mathrm{U}(\mathrm{eq})$ \\
\hline $\mathrm{C}(2)$ & $0.1903(2)$ & $0.8855(4)$ & $0.9464(2)$ & $0.028(4)$ \\
$\mathrm{C}(4)$ & -0.0222 & $1.0658(4)$ & $0.7826(4)$ & $0.028(4)$ \\
$\mathrm{N}(4)$ & $0.1424(2)$ & $0.9756(4)$ & $0.7947(4)$ & $0.030(4)$ \\
$\mathrm{N}(3)$ & $0.0184(4)$ & $0.8775(4)$ & $0.10380(4)$ & $0.026(4)$ \\
$\mathrm{N}(4)$ & -0.1401 & $0.7973(4)$ & $0.9684(4)$ & $0.031(4)$ \\
$\mathrm{N}(2)$ & $0.3009(2)$ & $0.9987(4)$ & $0.7068(4)$ & $0.033(4)$ \\
$\mathrm{O}(4)$ & -0.2706 & $0.7803(4)$ & $1.0621(4)$ & $0.043(4)$ \\
$\mathrm{O}(4)$ & $0.2701(2)$ & $1.0716(4)$ & $0.5779(4)$ & $0.049(4)$ \\
$\mathrm{O}(3)$ & -0.1406 & $0.7533(4)$ & $0.8161(4)$ & $0.048(4)$ \\
$\mathrm{O}(2)$ & $0.4628(4)$ & $0.9399(4)$ & $0.7609(2)$ & $0.047(4)$ \\
\hline
\end{tabular}

$\mathrm{x}, \mathrm{y}$ and $\mathrm{z}$ are the refined atomic coordinates in $\AA$ of the $\mathrm{x}, \mathrm{y}$ and $\mathrm{z}$ axis, respectively. $\mathrm{U}(\mathrm{eq})$ is the equivalent displacement parameter. Equivalent displacement parameters are in $\AA^{2}$.

and the difference with the real structure is less. In the subsequent calculation, the measured single crystal structure (structure A) will be taken as the original configuration. The differences between structure $\mathrm{A}$ and structure B can be caused by several reasons. The differences could be related to particle size effects, orientation of the HMX crystal and the test conditions (the 2-theta range $\left(3-65^{\circ}\right)$ may be too limited). All of these factors can affect the single crystal structure of HMX. We will study the specific reasons in the future work.
Table 2. The single crystal structure of HMX by experiment and from CCSD database.

\begin{tabular}{lcc}
\hline Parameters & Structure A (exp) & Structure B (CCSD) \\
\hline $\mathrm{a}\left(\right.$ Á$\left.^{\prime}\right)$ & $6.548(2)$ & 6.540 \\
$\mathrm{~b}\left({ }_{\AA} \mathrm{A}\right)$ & $11.041(4)$ & 11.050 \\
$\mathrm{c}\left({ }_{\AA}\right)$ & $7.369(3)$ & 8.700 \\
$\beta\left({ }^{\circ}\right)$ & 102.738 & 124.30 \\
$\rho\left(\mathrm{g} \cdot \mathrm{cm}^{-3}\right)$ & 1.893 & 1.8937 \\
Space group & $\mathrm{P} 21 / \mathrm{N}$ & $\mathrm{P} 21 / \mathrm{C}$ \\
Crystal system & Monoclinic & Monoclinic \\
$\mathrm{Z}$ & 2 & 2 \\
$\mathrm{R}$-factor/\% & 3.5 & 5.9 \\
\hline
\end{tabular}

\subsection{Growth morphology of HMX in vacuum}

The growth morphology of HMX in vacuum predicted by the AE model is shown in Figure 4. The morphology of HMX in vacuum is similar to a soccer ball and the crystal habit parameters of the main stable crystalline surfaces of HMX are listed in Table 3. The morphologically dominant faces are (011), (110), (020), (10-1) and (101), which account for 60.14, 30.90, 6.16, 1.76 and $1.04 \%$ of the total surface area, respectively. Among all the faces, the morphological importance of (011) is strongest for its largest $d_{011}(6.02 \AA)$ and largest face area (accounting for $60.14 \%$ of the total area). The attachment energy of $(011)$ is $-112.07 \mathrm{~kJ} \cdot \mathrm{mol}^{-1}$. The (101) face has the weakest morphological importance, accounting for $1.04 \%$ of the total face area. 


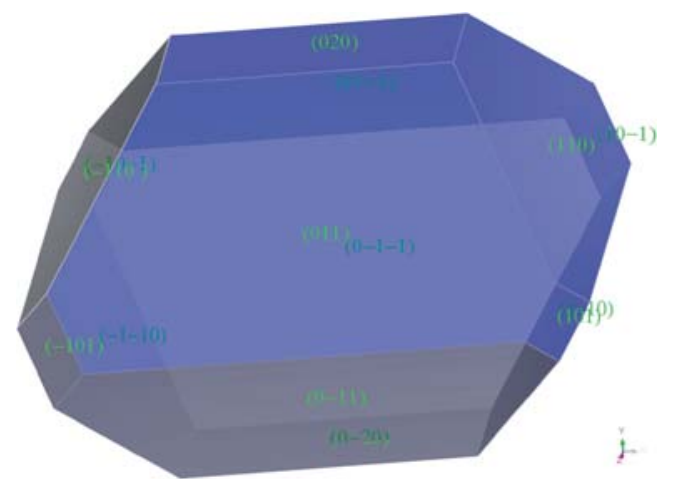

Figure 4. The predicted morphology of HMX by the AE model in vacuum.

\subsection{Growth morphology of HMX in acetone}

Before understanding the interaction between the HMX crystal surface and acetone molecules, it is meaningful to study the crystal surface of HMX. The molecular arrangments of different crystal surfaces are displayed in Figure 5. The surfaces of (110) and (10-1) are very rough. Surfaces (011), (020) and (101) are flat, especially (101). Paramater " $S$ " is introduced to describe the surface characteristics of the HMX crystal, which is the ratio of solvent accessible area to the corresponding
Table 4. The parameter $S$ values for the crystal habit surfaces of HMX.

\begin{tabular}{|c|c|c|c|c|c|}
\hline$(\mathrm{h} \mathrm{k} \mathrm{l})$ & $\left(\begin{array}{lll}0 & 1 & 1\end{array}\right)$ & $\left(\begin{array}{lll}1 & 1 & 0\end{array}\right)$ & $\left(\begin{array}{lll}0 & 2 & 0\end{array}\right)$ & $\left(\begin{array}{lll}1 & 0 & -1\end{array}\right)$ & $\left(\begin{array}{lll}1 & 0 & 1\end{array}\right)$ \\
\hline$A_{\mathrm{acc}}$ & 115.58 & 134.55 & 65 & 143.32 & 129.12 \\
\hline$A_{\mathrm{hkl}}$ & 86.92 & 94.60 & 48.25 & 96.19 & 120.13 \\
\hline$A_{\text {box }}$ & 782.28 & 851.34 & 434.27 & 865.70 & 1081.37 \\
\hline$S$ & 1.33 & 1.42 & 1.35 & 1.49 & 1.07 \\
\hline
\end{tabular}

All areas are in $\AA^{2}$. The solvent-accessible area of (h k l) faces in the unit cell. The surface area of the corresponding (h k l) face.

surface area. The calculated values of $A_{\mathrm{acc}}, A_{\mathrm{hkl}}$ and "S" are shown in Table 4. As shown in Table 4, all "S" values are in the range of 1-1.5. However, the differences of " $\mathrm{S}$ " values for the HMX crystal faces is very great. With the largest " $S$ " value (1.49), the $(10-1)$ face is very rough, which can promote the adsorption of solvent molecules. However, the "S" value of the (101) face is only 1.07. This small "S" value means morphological smoothness which does not facilitate to the adsorption of solvent. In addition, all 5 crystal faces have many of nitro groups exposed on the surface, whose polarity is very strong. The exposed nitro groups facilitate to the adsorption of acetone (Figure 6).

Table 3. The selected crystal habit parameters of HMX crystals in vacuum, predicted by the AE model.

\begin{tabular}{lccccc}
\hline (h $\mathrm{k}$ l) & Multiplicity & $d_{\mathrm{hkl}}$ & $E_{\text {att }}$ & $R_{\mathrm{hkl}}$ & Total face area/\% \\
\hline$\left(\begin{array}{lll}0 & 1 & 1\end{array}\right)$ & 4 & 6.02 & -112.07 & 1.00 & 60.14 \\
$\left(\begin{array}{lll}1 & 1 & 0\end{array}\right)$ & 4 & 5.53 & -167.32 & 1.49 & 30.90 \\
$\left(\begin{array}{lll}0 & 2 & 0\end{array}\right)$ & 2 & 5.52 & -162.23 & 1.45 & 6.16 \\
$\left(\begin{array}{lll}1 & 0 & -1\end{array}\right)$ & 2 & 5.40 & -219.37 & 1.96 & 1.76 \\
$\left(\begin{array}{lll}1 & 0 & 1\end{array}\right)$ & 2 & 4.32 & -186.85 & 3.26 & 1.04 \\
\hline
\end{tabular}

All energies are in $\mathrm{kJ} \cdot \mathrm{mol}^{-1}$, Interplanar distances are in $\AA$.

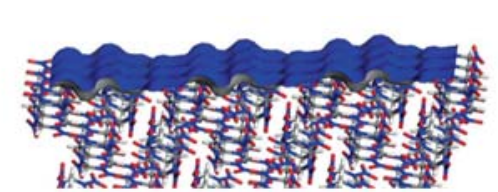

(011)

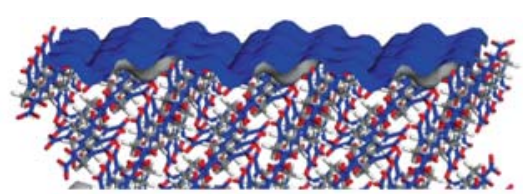

(110)

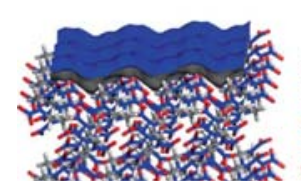

(020)

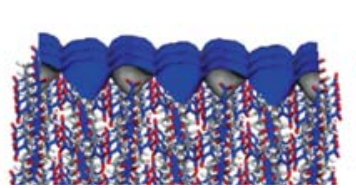

$(10-1)$

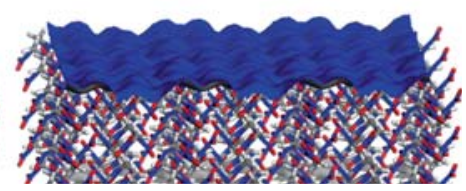

(101)

Figure 5. The molecular arrangement of different HMX crystal faces repesented by Connolly surfaces. ${ }^{38}$ 


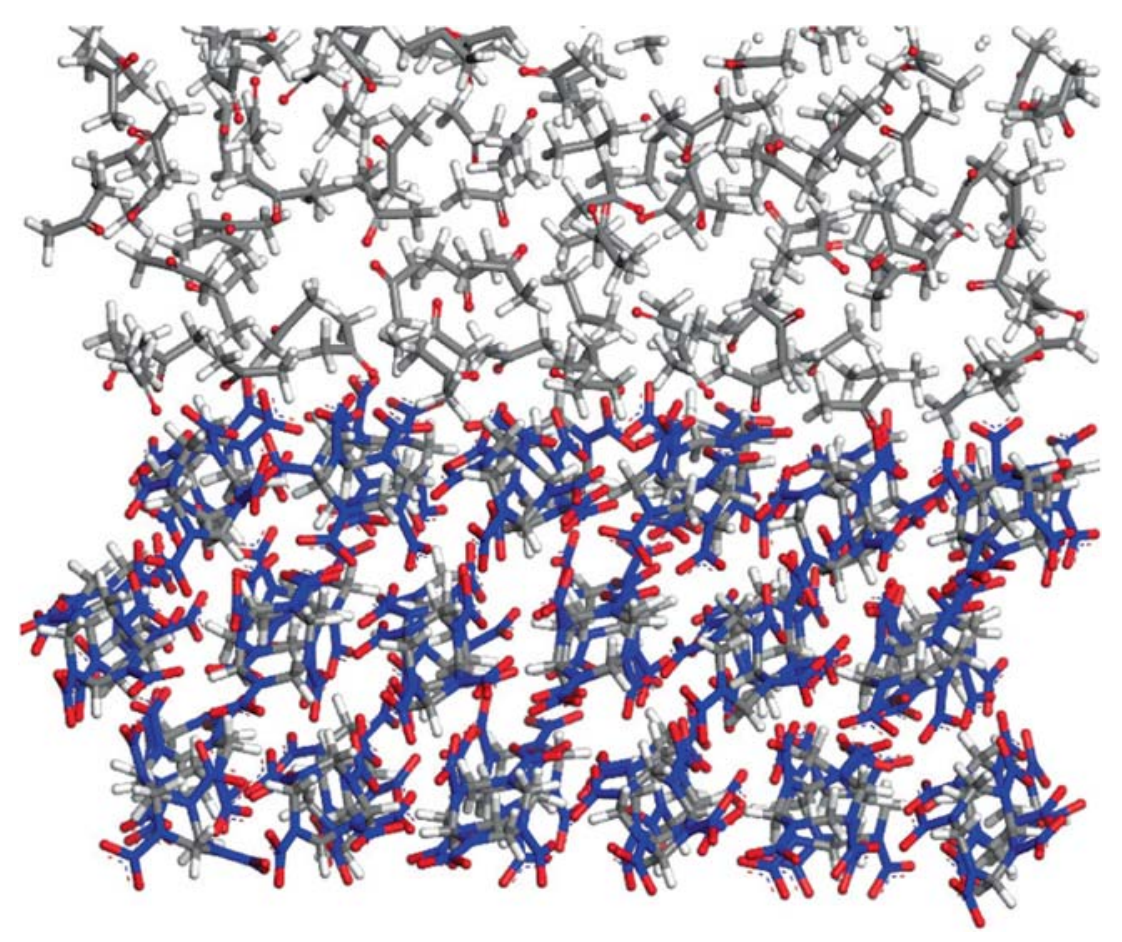

Figure 6. The equilibrium conformation $\operatorname{HMX}$ (110) face-acetone.

In order to understand the adsorption characteristics of acetone on different cystalline surfaces of HMX, the adsorption behavior was studied. Taking the (110) face as an example, acetone molecules and HMX molecules were found to be close together in the equilibrium conformation of the HMX-acetone system after performing molecular dynamics. It suggests that there are strong interactions between the acetone layer and the (110) face of HMX due to the strong polarity of acetone and the strong polarity of nitro groups exposed on the (110) face of HMX. The relative binding stength of acetone on different surfaces of HMX is as follows: $(10-1)>$ $(101)>(110)>(011)>(020)$. Besides the binding energy between the crystal surfaces and acetone, the contribution of van der Waals force and electrostatic force were calculated. As displayed in Table 5, the contribution of van der Waals force to the total binding energy of acetone layer with (011), (110), (020), (10-1) and (101) is 58.78, 55.44, 53.50, 55.42 and $63.85 \%$, respectively; the contribution of electrostatic force on the binding energy of an acetone layer with (011), (110), (020), (10-1) and (101) is 23.09, 32.41, 33.23,

Table 5. The binding energy between HMX crystalline surfaces (hkl) and acetone.

\begin{tabular}{lcrrrrr}
\hline \multirow{2}{*}{$\begin{array}{l}\text { Energy } \\
\text { types }\end{array}$} & $\begin{array}{c}\text { Energy } \\
\text { source }\end{array}$ & $(011)$ & $(110)$ & $(020)$ & \multicolumn{1}{c}{$\mathrm{E} /\left(\mathrm{kJ} \cdot \mathrm{mol}^{-1}\right)$} \\
\hline$E_{\text {total }}$ & $\mathrm{E}$ & -66394.91 & -66000.07 & -39609.26 & -66151.13 & -66161.58 \\
& $\mathrm{vdW}$ & -2780.58 & -2778.07 & -2760.85 & -3102.14 & -3241.59 \\
& Eelec & -72191.23 & -72055.26 & -44087.38 & -72233.91 & -72114.32 \\
$E_{\text {sur }}$ & $\mathrm{E}$ & -52498.38 & -51868.74 & -26129.01 & -52061.52 & -51902.81 \\
& $\mathrm{vdW}$ & -36.28 & 196.88 & 49.78 & 193.03 & 67.46 \\
& $\mathrm{Eelec}$ & -56170.38 & -55922.42 & -27962.28 & -56073.86 & -55824.82 \\
$E_{\text {solv }}$ & $\mathrm{E}$ & -13351.71 & -13376.58 & -12998.71 & -13018.65 & -13276.35 \\
& $\mathrm{vdW}$ & -2424.06 & -2556.49 & -2553.02 & -2701.66 & -2681.76 \\
& $\mathrm{Eelec}$ & -15895.08 & -15888.22 & -15965.09 & -15804.58 & -15975.75 \\
$E_{\text {inter }}$ & $\mathrm{E}$ & -544.82 & -754.74 & -481.54 & -1070.96 & -982.42 \\
& $\mathrm{vdW}$ & -320.23 & -418.46 & -257.61 & -593.52 & -627.29 \\
& Eelec & -125.78 & -244.61 & -160.01 & -355.48 & -313.75
\end{tabular}

Note: $E_{\text {total }}$ is the single-point energy of the equilibrium structure; $E_{\text {sur }}$ is the single-point energy of HMX; $E_{\text {solv }}$ is the singlepoint energy of acetone in the equilibrium structure; $E$ is the total energy of each structure; vdW is the energy of each structure obtained by the vdW interaction; $\mathrm{E}_{\text {elec }}$ is the energy of each structure obtained by electrostatic interaction. 
33.19 and $31.94 \%$, respectively. Therefore, HMX crystal surfaces interact with acetone layers almost in the same way, mainly via the van der Waals force.

After the analysis of the roughness of HMX crystal surfaces and the HMX-acetone interaction, the parameters for the crystal growth of HMX in acetone have been calculated. For acetone, the (10-1) face has the greatest solvent attachment energy $E_{\mathrm{s}}\left(-1070.96 \mathrm{~kJ} \cdot \mathrm{mol}^{-1}\right)$, and the (011) face has the lowest solvent attachment energy, only $-544.82 \mathrm{~kJ} \cdot \mathrm{mol}^{-1}$. The order of the solvent attachment energy for different crystal faces is as follows: $(10-1)>(101) \approx(110)>(011)>(110)$. The result suggests that the interaction intensity of the $(10-1)$ face with actone is greatest and the solvent adsorption capability of the (10-1) face is strongest, and the solvent adsorption capability of the (110) face is weakest. The adsorption capacity is mainly decided by two factors, the first is the number of the exposed polar groups on the crystal surface, and second is the roughness of the crystal surface (Table 6).

From the previous theory, interaction with an acetone layer will change the vacuum attachment energy of an HMX crystal surface. Therefore, the modified attachment energy was calculated by $E_{\text {att }}^{\prime}=E_{\text {att }}-E_{\mathrm{s}}$. As shown in Table 4, the modified attachment energy $\left(E_{\mathrm{att}}^{\prime}\right)$ of $(011)$ is lowest, only $-31.56 \mathrm{~kJ} \cdot \mathrm{mol}^{-1}$. The order of modified attachment energy for different crystal surfaces is: $(011)>(10-1)>(110)>(101)>$ (020). Therefore, the (011) face has great morphological importance for its slowest growth rate. Due to their fast growth rate, the (020) and (101) faces will disappear in the final crystal morphology of HMX.

Recrystallization of HMX in acetone was carried out. After recrystallization in acetone, the phase transition of $\beta$-HMX crystal did not happen. ${ }^{37-41}$ The crystal morphology of HMX before recrystallization is shown in Figure 7, the crystal morphology of HMX after recrystallization in Figure 8(a) and the predicted morphology is shown in Figure 8(b). The predicted morphology has a good consistency with SEM of HMX, both have a large (011) plane and the area ratio of each crystal is highly consistent. This suggests that accuracy and

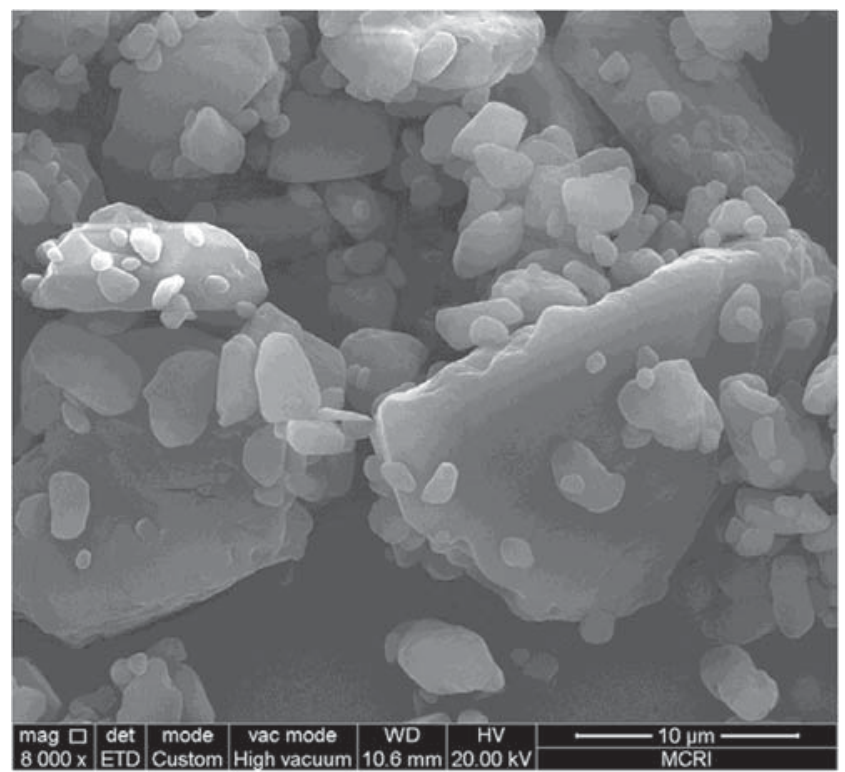

Figure 7. The crystal morphology of HMX before recrystallization.

reliability of using the original HMX structure and the attachment energy model to predict crystal growth morphology are high, in which the crystal surface roughness has been taken into account.

\subsection{Sensitivity}

Before recrystallization, the HMX crystal morphology is irregular and the particle size is variable. After recrystallization, the HMX crystal morphology is structured, polyhedron-shaped with a smooth surface. To study the influence of the recrystallization and crystal morphology on the sensitivity of HMX, the friction sensitivity and impact sensitivity of the samples before and after recrystallization were compared.

Before recrystallization, the shape and size of HMX crystals are irregular. The edges and sharp corners of the crystals are clear. In addition, due to the defect energy caused by the crystal defects, the crystals are more active. Thus, the sensitivity of the HMX before recrystallization

Table 6. Crystal growth parameters of HMX crystal habit faces in acetone.

\begin{tabular}{llcccrcc}
\hline Solvent & $(\mathrm{h} \mathrm{k} \mathrm{l})$ & $E_{\text {int }}$ & $A_{\text {acc }}$ & $A_{\text {box }}$ & \multicolumn{1}{c}{$E_{\mathrm{s}}$} & $E_{\text {att }}^{\prime}$ & $R_{\text {hkl }}^{\prime}$ \\
\hline Acetone & $(011)$ & -544.82 & 115.58 & 782.28 & -80.51 & -31.56 & 1.00 \\
& $(110)$ & -754.74 & 134.55 & 851.34 & -119.30 & -48.03 & 1.52 \\
& $(020)$ & -481.54 & 65 & 434.27 & -72.06 & -90.16 & 2.86 \\
& $(10-1)$ & -1070.96 & 143.32 & 865.70 & -177.32 & -45.10 & 1.43 \\
& $(101)$ & -982.42 & 129.12 & 1081.37 & -117.29 & -72.73 & 2.30 \\
\hline
\end{tabular}

All energies are in $\mathrm{kJ} \cdot \mathrm{mol}^{-1}$; distances are in $\AA$. 


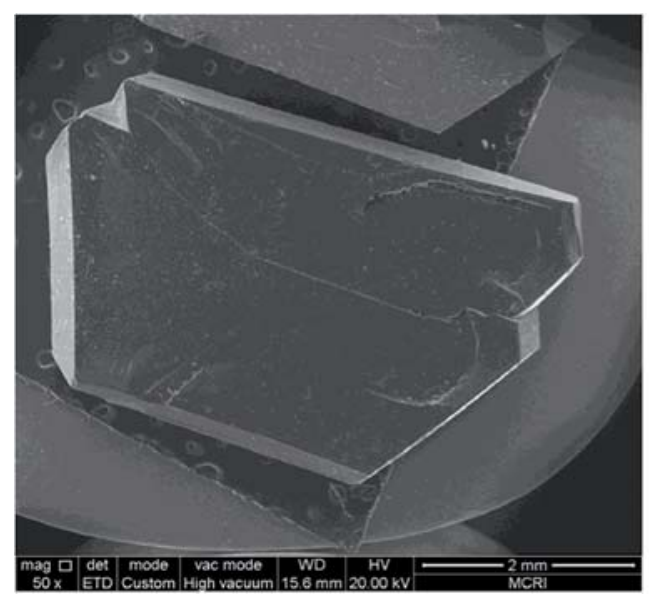

(a) SEM of HMX

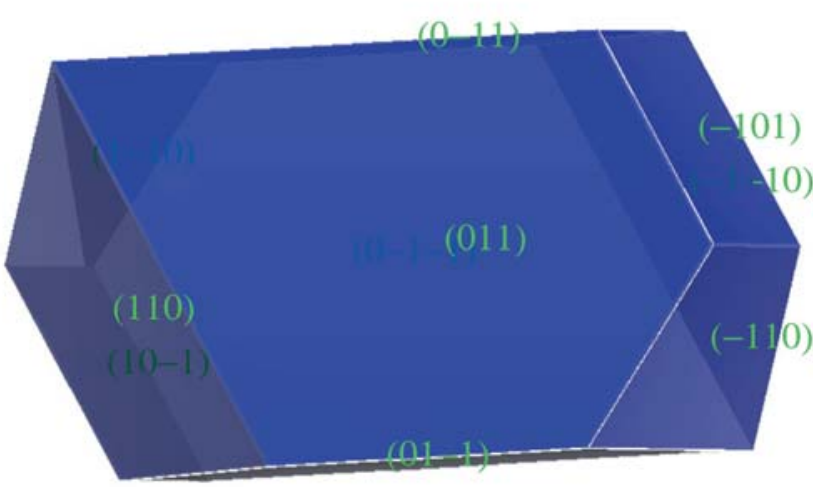

(b) predicted morphology

Figure 8. The crystal morphology of HMX after recrystallization.

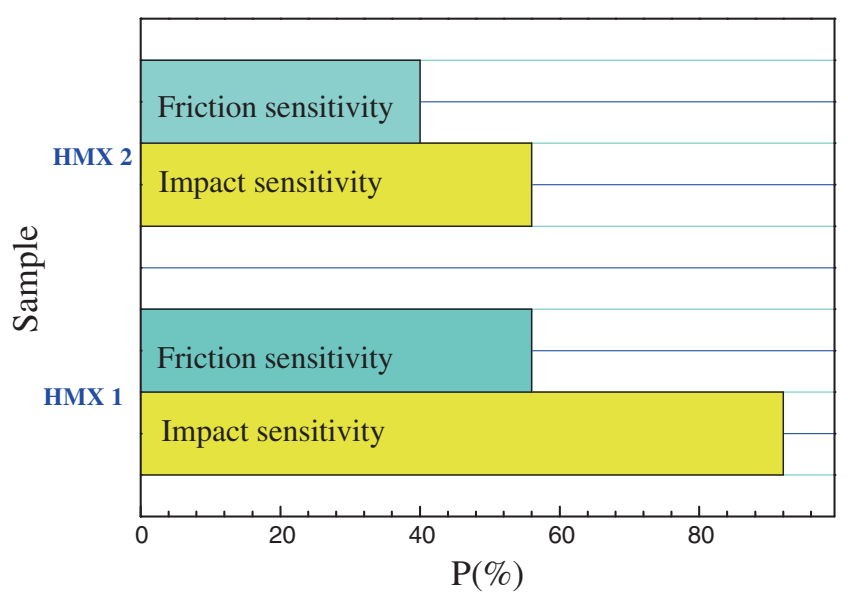

Figure 9. Sensitivities of two HMX samples. HMX1 and HMX 2 correspond to the crystals before and after recrystallization from acetone.

is high. After recrystallization, the crystal shape is a polyhedron and nearly-perfect. According to Bowden's hot spot theory, ${ }^{42}$ crystals with smooth surfaces can effectively reduce the risk of hot spots formed under mechanical force. In addition, the crystallographic perfection has a great effect on the sensitivity of explosive crystal. While the crystals are perfect and compact, the internal defects are few. In that case, there will be few hot spots, and the mechanical sensitivity is naturally lower than that of the HMX before recrystallization. As shown in Figure 9, recrystallization in acetone can effectively reduce the sensitivity of HMX, especially the impact sensitivity. After recrystallization, the impact sensitivity of HMX can be reduced from 92 to $64 \%$, a reduction of $28 \%$. These results effectively demonstrate that the sensitivity of explosives can be reduced by improving crystal morphology by recrystallization in acetone.

\section{Conclusions}

HMX single crystal was prepared and structure obtained. The attachment energy (AE) model was applied to predict the crystal morphology of HMX. Both the morphology and sensitivity of HMX before and after recrystallization were characterized. Specific conclusions are as follows: (4) HMX single crystal structure with $\mathrm{R}$ factor of 0.35 was obtained. The structure is different from the HMX crystal structures commonly used, which could be related to particle size effects, orientation of the HMX crystals and the test conditions. (2) The morphologically dominant faces are (011), (110), (020), (10-1) and (101). The order of binding of acetone on different surfaces of HMX is as follows: (10-1) $>(101)>(110)>(011)>(020)$. Crystal surfaces inter act with acetone layers mainly via van der Waals force, besides part electrostatic and other forces. (3) The (011) face is morphologically important for its slowest growth rate; (020) and (101) faces disappear in the final crystal morphology of HMX. (4) The predicted morphology is consistent with the SEM result. (5) Recrystallization in acetone can effectively reduce the sensitivity of HMX, especially the impact sensitivity.

\section{References}

1. Hamshere B L, Lochert I J and Dexter R M 2003 In Evaluation of PBXN-109: The explosive fill for the Penguin Anti-Ship Missile Warhead. K Gooley (Ed.) (Edinburgh: Defence Science and Technology Organisation Salisbury Systems Sciences Lab) p. 27

2. Kim K J, Park J H and Kim J B 2013 A Numerical Study on the Velocity of a Cylindrical Counter Projectile Using LX-14 Explosive Appl. Mech. Mater. 42146

3. Hoffman D M 2000 Fatigue of LX-14 and LX-19 Plastic Bonded Explosives J. Energ. Mater. 181 
4. Wu Y G, Wu X Q, Chen H W, Zhang L and Zhang C 2009 Performance of Nitramine Propellants with Different Phases of HMX Chin. J. Energ. Mater. 17206

5. Borne L 1998 Explosive Crystal Microstructure and Shock-Sensitivity of Cast Formulations Proc. 11th Symposium (International) on Detonation, Snowmass, CO, August 30-September 4, 1998, p. 658

6. Antoine E D M and van der Heijden 2004 Physicochemical Parameters of Nitramines Influencing Shock Sensitivity Propellants Explos. Pyrotech. 29304

7. Kaully T and Keren B 2000 Paste explosive based on rouned HMX: Rheology, sensitivity, and mechanical properties Insensitive Munitions \& Energetic Materials Technology Symposium. San Antonio, Teas

8. Huang H J, Dong H S and Shu Y J 2003 The preparation of HMX crystals with defects and the influences of crystal defects on thermal sensitivity and stability Energy Mater. 11123

9. Czerski H, Greenaway M W, Proud W G and Field J E $2004 \beta-\delta$ phase transition during dropweight impact on HMX J. Appl. Phys. 964131

10. Maruyama S and Ooshima H 2001 Mechanism of the solvent-mediated transformation of taltirelin polymorphs promoted by methanol Chem. Eng. J. 811

11. Hod I, Mastai Y and Medina D D 2011 Effect of solvents on the growth morphology of DL-alanine crystals CrystEngComm 13502

12. Sangwal K, Zdyb A, Chocyk D and Mielniczek-Brzóska E 1996 Effect of Supersaturation and Temperature on the Growth Morphology of Ammonium Oxalate Monohydrate Crystals Obtained from Aqueous Solutions Cryst. Res. Technol. 31267

13. Horst J H T, Geertman R M, Heijden A E V D and Rosmalen G M V 1999 The influence of a solvent on the crystal morphology of RDX J. Cryst. Growth 198773

14. Chen J and Trout B L 2010 Computer-aided solvent selection for improving the morphology of needle-like crystals: A case study of 2,6-dihydroxybenzoic acid Cryst. Growth Des. 104379

15. Shim H M and Koo K K 2014 Crystal Morphology Prediction of Hexahydro-1,3,5-trinitro-1,3,5-triazine by the Spiral Growth Model Cryst. Growth Des. 141802

16. Gavezzotti A 2013 Crystal formation and stability: Physical principles and molecular simulation Cryst. Res. Technol. 48793

17. ter Horsta J H, Geertmanb R M and van Rosmalena G M 2001 The effect of solvent on crystal morphology J. Cryst. Growth 230277

18. Shi W Y, Xia M Z, Lei W and Wang F Y 2014 Solvent effect on the crystal morphology of 2,6-diamino3,5-dinitropyridine-1-oxide: A molecular dynamics simulation study J. Mol. Graphics Modell. 5071

19. Liu N, Li Y N, Zeman S, Shu Y J, Wang B Z, Zhou Y S, Zhao Q L and Wang W L 2016 Crystal morphology of 3,4-bis (3-nitrofurazan-4-yl) furoxan (DNTF) in a solvent system: Molecular dynamics simulation and sensitivity study CrystEngComm 182843

20. Liu N, Wang B Z, Shu Y J, Wu Z K, Zhou Q, Zhao Q L and WANG W L 2016 Molecular Dynamics Simulation on Crystal Morphology of FOX-7 Chin. J. Explos. Propell. 3940

21. Ballav M B, Bhaskar J B and Gopal D 2006 Lowmolecular-weight poly-carboxylate as crystal growth modifier in biomineralization J. Chem. Sci. 118519
22. Bagchi B and Kirkpatrick T R 1986 On the kinetics of crystal growth from a supercooled melt J. Chem. Sci. 96465

23. Berkovitch-Yellin Z 1985 Toward an ab initio derivation of crystal morphology J. Am. Chem. Soc. 1078239

24. Hartman P 1968 Theoretical morphology of crystals with the SnI 4 structure J. Cryst. Growth 2385

25. Tao J and Wang X F 2017 Molecular dynamics simulation for fluoropolymers applied in $\varepsilon$-CL-20-based explosive J. Adhes. Sci. Technol. 31250

26. Material Studio 3.0, Accelrys, San Diego, Ca, 2004

27. Lorenzo L D, Tocci E, Gugliuzza A, Macchione M and Drioli E 2012 Pure and modified co-poly (amide-12-bethylene oxide) membranes for gas separation studied by molecular investigations Membranes 2346

28. Connolly M L 1983 Solvent-accessible surfaces of proteins and nucleic acids Science 221709

29. Sun H 1998 COMPASS: An ab initio force-field optimized for condensed-phase applications-Overview with details on alkane and benzene compounds J. Phys. Chem. B 1027338

30. Tao J, Wang X F, Zhao S X, Wang C L, Diao X Q and Han Z X 2017 Simulation and calculation for binding energy and mechanical properties of $\varepsilon$-CL-20/energetic polymer binder mixed system Chin. J. Energy Mater. 23315

31. Tao J, Wang X F, Zhao S X, Diao X Q, Wang C L and Han Z X 2016 Molecular dynamics simulation of CL-20/ HMX cocrystal and blends Chin. J. Energy Mater. 24324

32. Palmer S J P, Field J E and Huntley J M 1993 Deformation, Strengths and Strains to Failure of Polymer Bonded Explosives Proc. R. Soc. London, Ser. A 440399

33. Jaidann M, Abou H, Lafleur X and Brisson J 2011 Atomistic studies of RDX and FOX-7-Based PlasticBonded explosives: molecular dynamics simulation Procedia Comput. Sci. 41177

34. Jin B, Shen J, Peng R F, Shu Y J, Chu S J and Dong H S 2012 Synthesis, characterization, thermal stability and mechanical sensitivity of polyvinyl azidoacetate as a new energetic binder J. Polym. Res. 199974

35. Jennifer J and Michel P 2001 Particle design using supercritical fluids: Literature and patent survey $J$. Supercrit. Fluids 20179

36. Lee B M, Kim J S, Lee B C, Kim H S, Kim H and Lee Y W 2011 Preparation of micronized $\beta$-HMX using supercritical carbon dioxide as antisolvent Ind. Eng. Chem. Res. 509107

37. Jiang Y L, Xu J J, Zhang H B and Sun J 2013 Research progress on HMX crystallization morphology Mater. Rev. 2711

38. Boissonnat J D, Devillers O, Duquesne J and vinec M Y 1994 Computing connolly surfaces J. Mol. Graphics 1261

39. Kim C K, Lee B C and Lee Y W 2009 Solvent effect on particle morphology in recrystallization of HMX (cyclotetramethylenetetranitramine) using supercritical carbon dioxide as antisolvent Korean J. Chem. Eng. 261125

40. Bowden F P, Yoffe A D and Hudson G E 1952 Initiation and Growth of Explosions in Liquids and Solids Am. J. Phys. 20250

41. Coffey C S and Armstrong R W 1981 In Description of "hot spots" associated with localized shear zone $s$ in impact tests, shock waves and high strain rate phenomena in metals L David (Ed.) (New York: Plenum Press)

42. Thomas P H 1965 A comparison of some hot spot theories Combust. Flame $\mathbf{9} 369$ 